\title{
Modelagem de perfis geofísicos sintéticos em poços da Bacia do Recôncavo
}

Ariadne Amaro Nicácio ${ }^{1}$ da Silva, Breno Figueiredo Bahia ${ }^{1}$, Taíla Crístia Souza Sant'ana ${ }^{1}$, Vinícius Carneiro Santana ${ }^{2}$ e Michael Holz ${ }^{3}$

${ }^{1}$ Graduação em Geofísica - UFBA, ${ }^{2}$ Pós Graduação em Geofísica - UFBA, ${ }^{3}$ Professor adjunto - UFBA

Copyright 2014, SBGf - Sociedade Brasileira de Geofísica

Este texto foi preparado para a apresentação no VI Simpósio Brasileiro de Geofísica, Porto Alegre, 14 a 16 de outubro de 2014. Seu conteúdo foi revisado pelo Comitê Técnico do VI SimBGf, mas não necessariamente representa a opinião da SBGf ou de seus associados. É proibida a reprodução total ou parcial deste material para propósitos comerciais sem prévia autorização da SBGf.

\section{Resumo}

Perfis geofísicos são utilizados nas atividades de exploração e explotação de hidrocarbonetos, não apenas na quantificação de propriedades físicas de reservatórios, mas também no campo da Interpretação Sísmica. Os perfis sônico (DT) e densidade (RHOB), por exemplo, são utilizados na calibração sísmica/poço (Meireles, Pinto, Nery, 2012), e sua ausência constitui um problema para o intérprete, visto que, a partir deles, seria possível a confecção de sismogramas sintéticos. Poços exploratórios mais antigos acompanham este problema, pois a perfilagem era realizada apenas nos intervalos de interesse. Já bastante difundidos na literatura, alguns métodos de modelagem podem sintetizar essas propriedades. Equações empíricas e métodos estatísticos podem ser utilizadas com o intuito de modelar os perfis de interesse. O presente trabalho tem como objetivo comparar modelagens realizadas a partir das equações empíricas em poços da Bacia do Recôncavo, como a equação de Wyllie, e futuramente a partir de métodos estatísticos, para definir a melhor maneira de sintetização das propriedades petrofísicas úteis para a confecção de sismogramas sintéticos.

\section{Introdução}

A correlação sísmica/poço desempenha um papel muito importante na interpretação sísmica. Com esta, o intérprete adiciona certeza e confiabilidade ao seu trabalho. Entretanto, os passos realizados no processo de "amarração" entre o dado sísmico e os dados do poço podem ser turbulentos. A amarração entre sísmica e poço é realizada a partir da confecção de um sismograma sintético, produto da convolução de uma wavelet com a função refletividade.

Para se obter um sismograma sintético confiável, necessita-se da wavelet que mais se aproxime da assinatura da fonte e da função refletividade que represente a geologia em subsuperfície de maneira precisa. A obtenção da wavelet não é o foco deste trabalho. A função refletividade é baseada no contraste de impedância acústica das camadas, sendo a impedância acústica definida como o produto entre a velocidade da onda sonora na camada pela densidade da mesma.

A obtenção dos valores correspondentes a estas propriedades físicas é feita a partir dos perfis geofísicos de densidade (RHOB) e sônico (DT). Em condições ideais, os dois perfis básicos para a confecção do sismograma sintético seriam "corridos" em todo o poço, entretanto, especialmente para poços antigos, isto não ocorre. Antigamente, apenas os intervalos de interesse eram perfilados, o que constitui um problema na amarração sísmica/poço.
De acordo com Bucheb e Rodrigues (1997), admite-se que uma curva de perfil pode ser considerada função de outras variáveis, ou seja, as outras curvas de perfis. Então, o objetivo é modelar densidades e tempos de trânsito em poços onde esses dados reais existem para que possam ser comparados e, após a comparação, expandir esses resultados para poços em que não haja esses dados.

Uma das formas de se alcançar este objetivo é a aplicação de equações empíricas. Uma delas, a equação de Gardner et all (1974), é largamente utilizada na tentativa de se obter as densidades das camadas a partir das velocidades de propagação das ondas sonoras nestas. A utilização indiscriminada das equações empíricas pode não ser eficiente, uma vez que cada uma delas é direcionada a uma certa região, onde o respectivo autor realizou o seu trabalho. Isto, infelizmente, adiciona erros à modelagem numérica baseada neste tipo de equações. Não apenas a equação proposta por Gardner et. all (1974) está susceptível a este fardo, já que todas as outras equações empíricas utilizadas neste trabalho baseiamse em constantes, as quais se adaptam à região onde foi realizado o estudo do autor, mas não necessariamente à região do presente trabalho. As condições do poço e do reservatório também são extremamente importantes, tendo em vista que estas condições afetarão as medições das propriedades físicas que serão utilizadas nas equações empíricas. Logo, poços com boa integridade serão essenciais para uma melhor modelagem. Entretanto, a integridade do poço pode estar comprometida, a exemplo de desmoronamentos, e isto afeta medida de algumas propriedades.

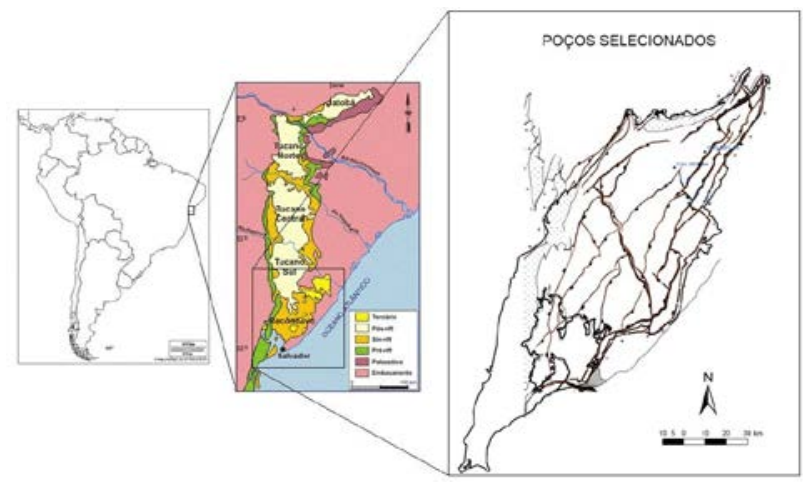

Figura 1 - Localização dos poços utilizados no trabalho, na Bacia do Recôncavo, nordeste do Brasil. 


\section{POÇOS SELECIONADOS}

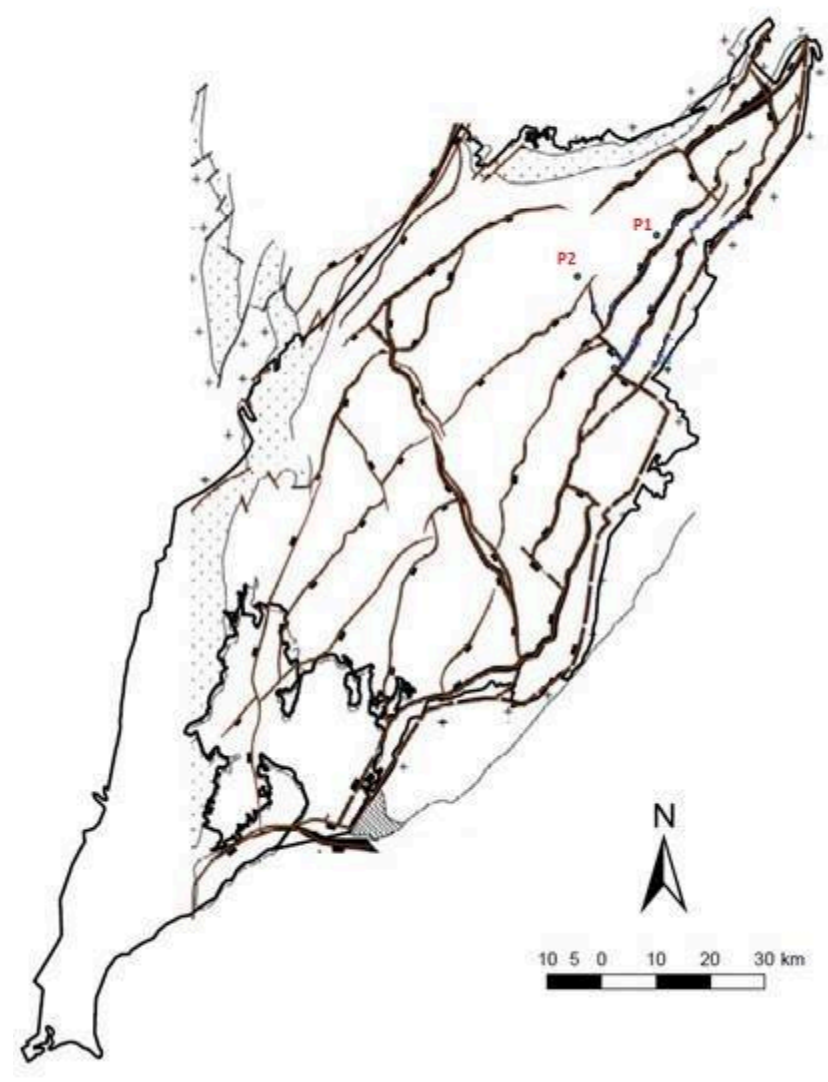

Figura 2 - Localização dos poços utilizados

\section{Metodologia}

Como as medidas dos perfis geofísicos estão susceptíveis a variações devido às condições do poço, diferentes intervalos foram escolhidos para evidenciar a influência destas condições sob a modelagem. A presença da curva que traduz a integridade do poço (Cáliper) foi de crucial importância nesta fase da pesquisa. Procurou-se, também, a presença de outras curvas, como as de resistividade, gamma-ray e potencial espontâneo, as quais darão suporte às modelagens com base em equações empíricas e em métodos baseados em análise estatística.

A pesquisa está dividida em duas fases; a primeira refere-se à modelagem com base em equações empíricas bastante comuns na literatura, como as de Wyllie et. all (1956), Raymer et. all (1980), Smits (1968) e Gardner et. all (1974), cujas análises estão presentes neste trabalho. A segunda fase (em andamento), por sua vez, faz referência à modelagem com métodos baseados em análise estatística, os quais já se mostraram muito promissores, como evidenciado nos trabalhos de Pinto (2011), Meireles (2011), Santos (2010) e Meireles et all. (2012). Para realização do trabalho, foram escolhidos 2 poços da região central da Bacia do Recôncavo, aos quais nos referimos como poço 1 e poço 2, mostrados na Figura 1. Perfis de tempo de trânsito e densidade foram modelados com as equações empíricas citadas anteriormente e os resultados foram comparados com as curvas originais. Nos poços 1 e 2 estão disponíveis as curvas de Cáliper (CAL), Gamma Ray (GR), Indução profunda (ILD), Densidade (RHOB) e Sônico (DT). Além destes, o poço 1 possui o perfil de porosidade NPHI.

Espera-se, para um caso ideal, que a modelagem de uma certa propriedade física, para um certo ponto, resulte no valor lido pela ferramenta, ainda que submetido a péssimas condições ambientais na perfilagem. Em função disto, o coeficiente de correlação linear $\left(R^{2}\right)$ entre os valores modelados (sintéticos) e os valores originais da perfilagem deveria, no caso ideal, ser igual à unidade. A obtenção do coeficiente de correlação foi realizada no software Microsoft Office Exce/ ${ }^{\circledR}$, a partir da linha de tendência entre os pontos citados.

O coeficiente de correlação indica qual equação obteve melhores resultados, mas, para uma melhor análise, os perfis sintético e original são dispostos de maneira similar ao que seria visto no poço, viabilizando a análise da forma das curvas. De maneira similar, devese analisar o efeito de desmoronamentos e estrangulamentos no poço. Para tanto, a curva Cáliper é posta lado a lado às curvas sintéticas e original, com o intuito de expor desvios e erros relacionados às condições do poço.

Para o caso do DT, modelos Tempo-Profundidade são gerados para cada curva modelada com o intuito de avaliar qual equação gerou resultados mais promissores.

Com a análise destes resultados será possível escolher qual equação é a mais adequada para modelagem dos perfis em questão.

Como já dito, as seguintes equações foram utilizadas:

1. Equação do tempo médio de Wyllie et all. (1956);

2. Equação de Raymer et all. (1980);

3. Equação de Gardner et all. (1974), e

4. Equação de Smits (1968).

As equações citadas acima relacionam as seguintes propriedades, respectivamente:

1. Porosidade com o tempo de trânsito das ondas compressionais nas partes de um sistema - matriz mais poro ou o que está preenchendo-o.

2. Porosidade com o tempo de trânsito das ondas compressionais nas partes de um sistema, já que é uma modificação da equação de Wyllie.

Os valores de tempo de trânsito e densidade utilizados para esses 2 casos foram aqueles mais empregados pela indústria: $\Delta \mathrm{t}_{\text {fluido }}=189$ $\mu \mathrm{s} /$ pé e $\Delta \mathrm{t}_{\text {matriz }}=55,5 \mu \mathrm{s} /$ pé, $\rho_{\mathrm{f}}=1,0 \mathrm{~g} / \mathrm{cm}^{3} \mathrm{e}$ $\rho_{\mathrm{m}}=2,65 \mathrm{~g} / \mathrm{cm}^{3}$.

3. A velocidade da onda $P$ na rocha com a porosidade. 
4. Resistividade/condutividade com tempo de trânsito.

\section{Resultados}

As equações empíricas utilizadas foram elaboradas para modelos específicos, então os resultados não serão os mesmos para o caso estudado nesse trabalho, como a equação do tempo médio de Wyllie et al. (1956) (1):

$$
\Delta t=\Phi \cdot \Delta t_{\text {fluido }}+(1-\Phi) \cdot \Delta t_{\text {matriz }}
$$

(1)

Segundo Mavko et all. (2009), esta equação é utilizada para o caso de rochas isotrópicas, de mineralogia uniforme e $100 \%$ saturadas por água. Para rochas consolidadas com porosidade primária e intermediária, ou seja, para porosidades muito altas deve-se buscar outros modelos. Para essa equação podemos utilizar o perfil de porosidade NPHI, quando existir, e RHOB para obter os tempos de trânsito.

A equação de Raymer et all (1980) (2) possui as mesmas suposições e limitações da equação de Wyllie supondo sempre a porosidade $<37 \%$.

$\Delta \mathrm{t}=\left[\frac{\Phi}{\Delta \mathrm{t} \text { fluido }}+\frac{(1-\Phi)^{2}}{\Delta \mathrm{tmatriz}}\right]^{-1}$

É importante ressaltar que, apesar de a equação de Raymer ser uma modificação da equação de Wyllie, elas ainda são diferentes, então pode haver dados que se encaixem melhor com uma do que com a outra. Pode-se usar os mesmos perfis de porosidade citados acima.

$$
\Delta t=91 R_{t}^{-0,15}
$$

A equação de Gardner et al (1974) (3), segundo Mavko et al. (2009), expressa uma média para vários tipos de rocha, ou seja, o caso atual pode ajustar-se ora melhor ora pior para essa equação. É largamente utilizada na sísmica, e como relaciona a velocidade das ondas compressionais com a densidade por razão inversa, pode ser usada para relacionar o tempo de trânsito das ondas compressionais com a densidade. Pode-se utilizar o perfil de densidade RHOB para a modelagem do DT, como mostrado na equação (4), ou vice-versa.

$$
\Delta t=\left[\frac{0.23}{\rho_{b}}\right]^{4}
$$

A equação de Smits (1968) (4), por sua vez, propõe um modelo físico que descreve a sua equação, sendo assim, é específica para os dados do seu trabalho, podendo ou não se ajustar aos dados presentes. Para a aplicação, é necessário entrar com o valor da resistividade da formação, então pode-se usar o perfil de Indução Profunda (ILD).

A figura 3 mostra o perfil cáliper ao lado do perfil sônico original no intervalo estudado (1950m a $3330 \mathrm{~m})$ do poço 1. O perfil cáliper, que mede o diâmetro do poço, é usado como avaliador da integridade do poço ou qualidade da sua perfuração, portanto reflete diretamente nas qualidades das curvas dos perfis, isto é, em intervalos com desmoronamentos a medição de outros perfis não apresentam uma boa confiabilidade.

Podemos observar na Figura 4 a relação de dispersão entre os perfis sônico original e o sônico sintético gerado a partir da equação de Raymer et al. (1980) para o poço 1. Entre as equações empíricas utilizadas para a modelagem das curvas de tempo de trânsito, a equação de Raymer et al. (1980) utilizando o perfil $\mathrm{NPHI}$ foi a que apresentou um melhor resultado. Foi obtido um coeficiente de correlação $\left(R^{2}\right)$ de valor igual a 0,7195, que é considerado satisfatório; isso significa que os pontos das curvas desses dois perfis estão mais próximos e, portanto eles são mais semelhantes.

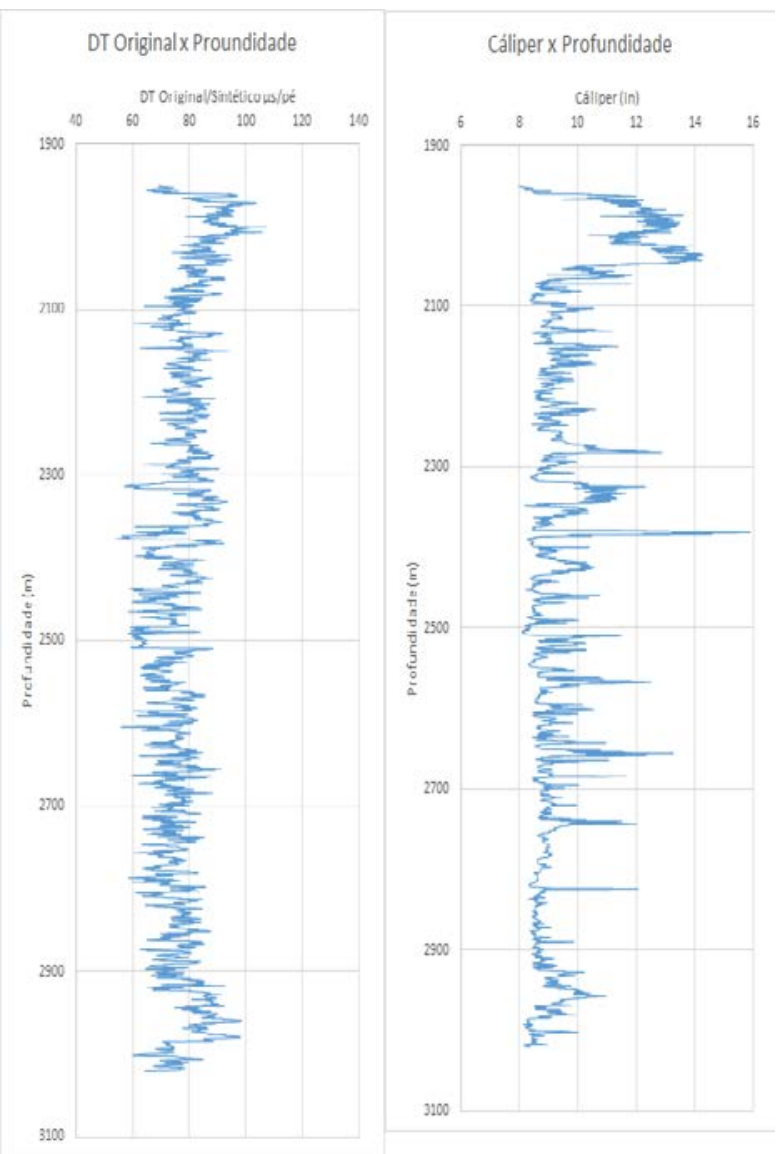

Figura 3 - Perfil Cáliper ao lado do sônico original no intervalo do poço 1. 


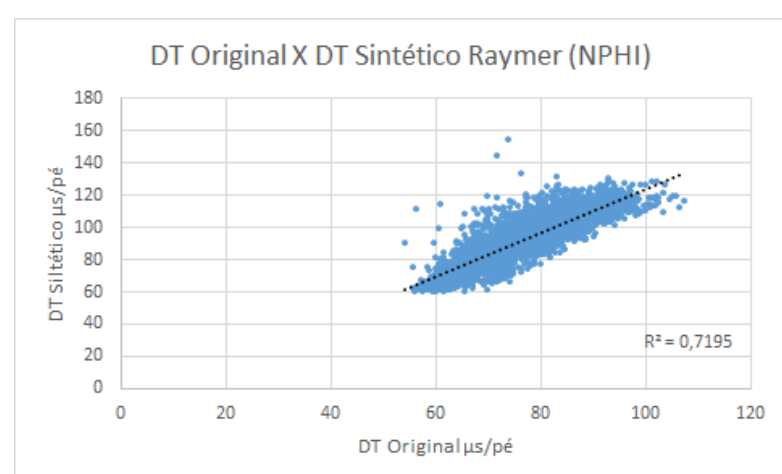

Figura 4 - Relação de dispersão entre os perfis sônicos original e sintético modelado a partir da equação de Raymer et al.(1980) utilizando NPHI para o poço 1.

Na figura 5 estão plotados os perfis sônicos original e sintético para o poço 1. Nos intervalos do perfil cáliper em que o poço se apresenta desmoronado, é possível observar que o tempo de trânsito atinge valores maiores, fato observado tanto na curva original quanto na curva sintética. Nota-se também que o pico presente no perfil cáliper por volta de $2400 \mathrm{~m}$ de profundidade, é refletido em ambos perfis sônicos. Conclui-se assim, que, apesar de os perfis não serem iguais, eles são correspondentes.

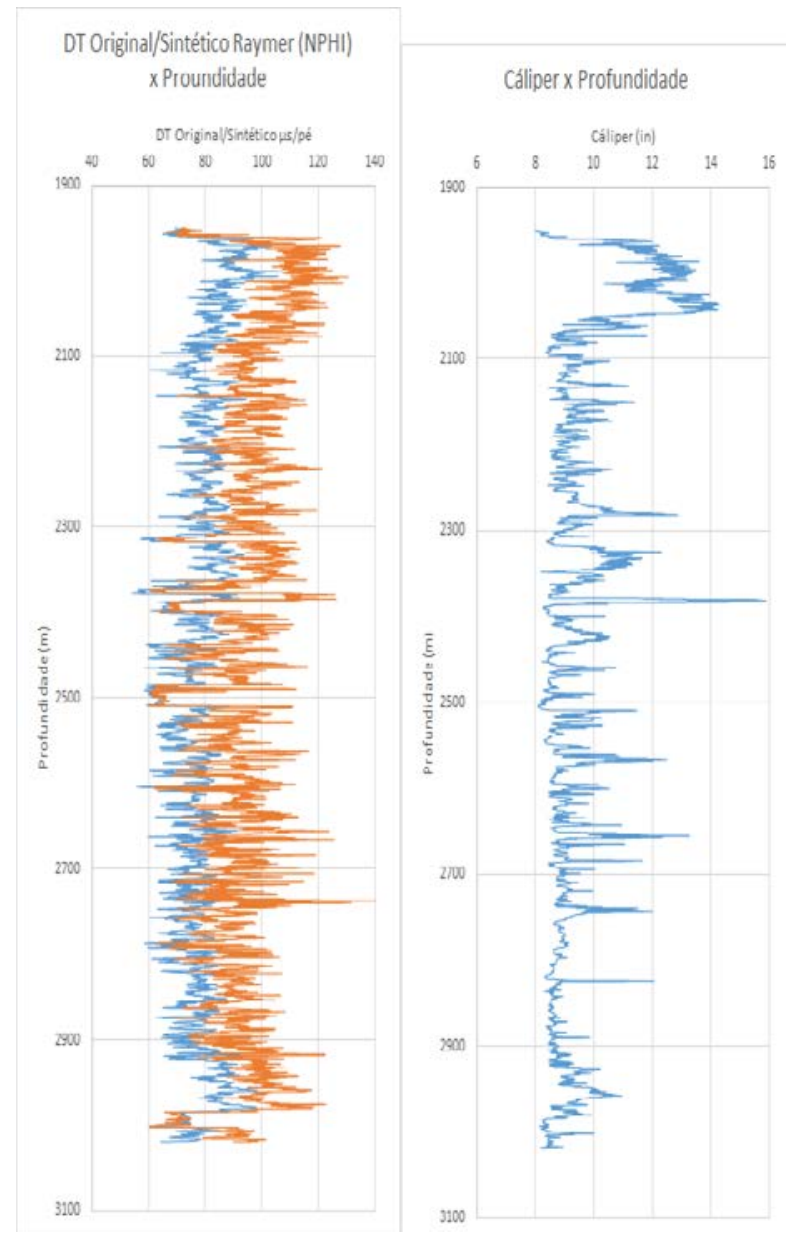

Figura 5 - Relação entre os perfis sônicos original e sintético modelado a partir da equação de Raymer et al.(1980) utilizando NPHI, ao lado do Cáliper, utilizando dados do poço 1.
Foram elaboradas curvas de Tempo x Profundidade através dos perfis sônicos sintéticos gerados por todas as equações empíricas citadas anteriormente. A figura 6 ilustra as curvas Tempo x Profundidade original e sintética a partir da equação de Smits (1968) para o poço 1 , sendo que esta última foi a que mais se aproximou da curva original. Essas curvas foram elaboradas com 0 intuito de futuramente gerar sismogramas sintéticos e assim proporcionar aos poços que não contém as curvar necessárias, a geração dos mesmos.

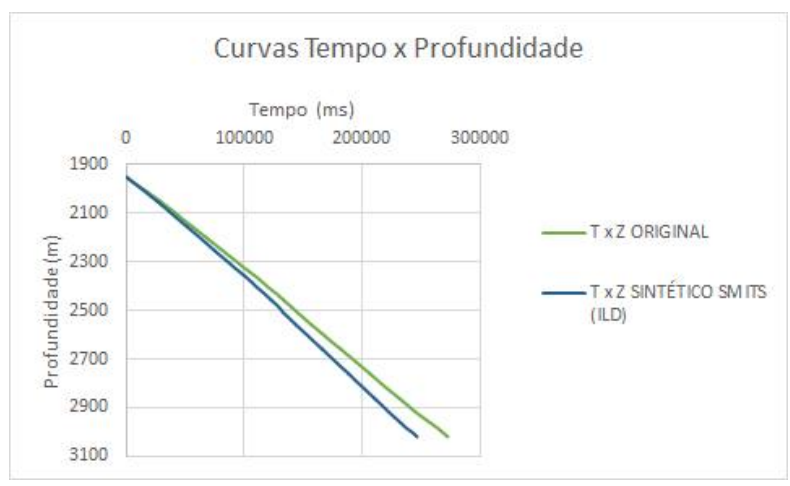

Figura 6 - Curvas Tempo x Profundidade original e sintética a partir da equação de Smits (1968) para o poço 1.

A figura 7 mostra o perfil cáliper ao lado do perfil sônico original no intervalo estudado do poço 2 (2550m a $3000 \mathrm{~m})$. É possível observar novamente relação entre a qualidade da perfuração do poço e dos perfis corridos. 


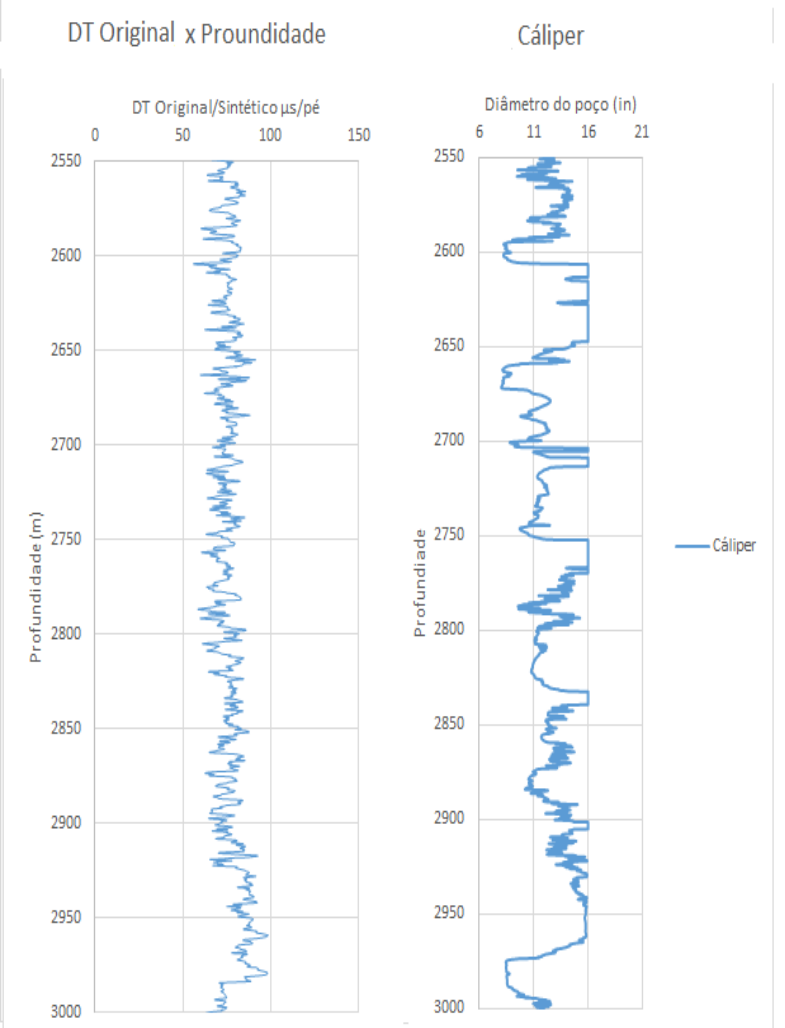

Figura 7 - Relação entre os perfis sônicos original e sintético modelado a partir da equação de Gardner (1974) ao lado do Cáliper para o poço 2.

Na figura 8 está ilustrada a relação de dispersão entre os perfis sônicos original e sintético modelado através da equação empírica de Gardner (1974), que foi a que melhor se ajustou para o poço 2.

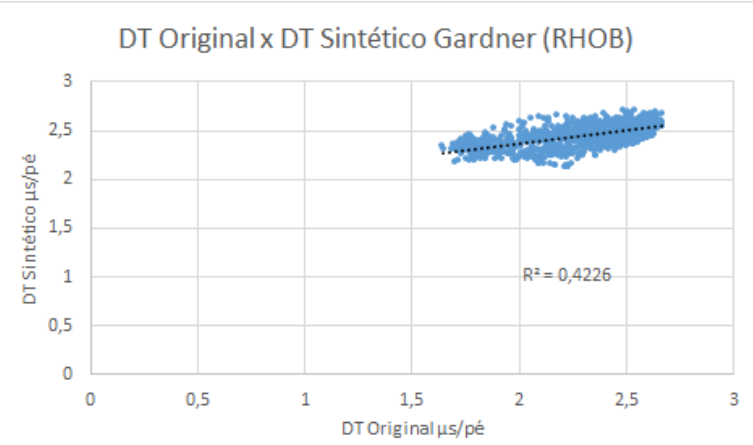

Figura 8 - Relação de dispersão entre os perfis sônicos original e sintético modelado a partir da equação de Gardner et al.(1974) no caso do poço 2.

A figura 9 mostra os perfis sônicos original e sintético plotados e comparados. E possível notar que em intervalos mais desmoronados no perfil do Cáliper, a diferença entre os perfis sônicos é mais acentuada. Portanto, uma menor confiabilidade no perfil sônico original é refletida na curva sintética modelada.

\section{DT Original/Sintético Gardner \\ (RHOB) $x$ Proundidade}
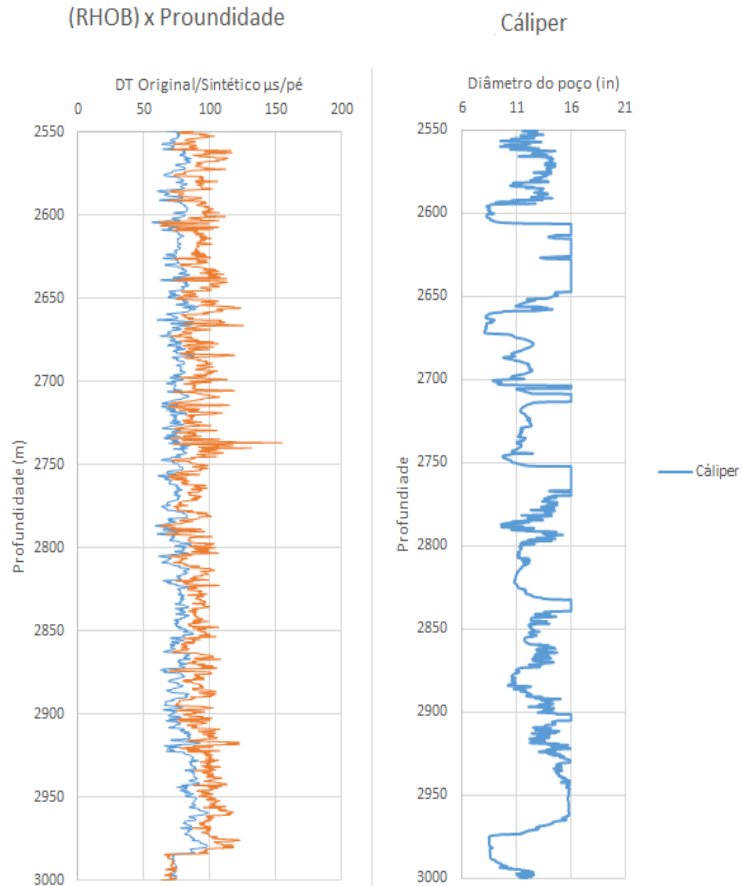

Figura 9 - Relação entre os perfis sônicos original e sintético modelado a partir da equação de Gardner (1974), ao lado do Cáliper, para o poço 2.

Na figura 10 vê-se as curvas de Tempo x Profundidade geradas a partir dos perfis sônicos original e sintético modelado através da equação de Wyllie et al (1956), que apresentou uma maior proximidade para o poço 2 .

\section{Curvas Tempo x Profundidade}

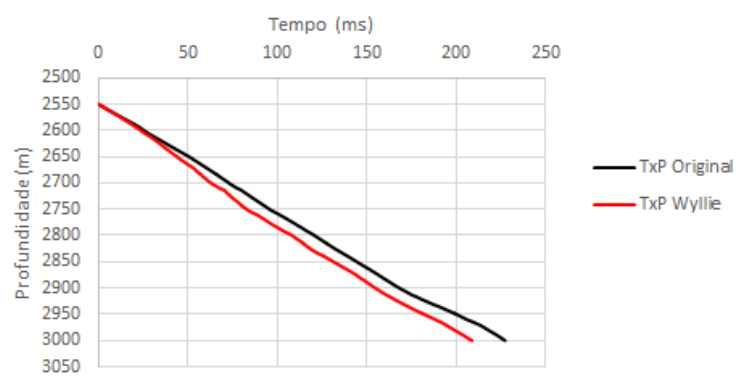

Figura 10 - Curva de Tempo x Profundidade original e sintética a partir da equação de Wyllie et al (1956) para o poço 2 .

\section{Discussão e Conclusões}

Ainda que para a equação de Raymer et al (1980) a partir do perfil de porosidade NPHI no poço 1 , o coeficiente de correlação tenha sido satisfatório, este resultado não ocorreu com muita frequência para as outras equações empíricas. Alguns apresentaram coeficientes de correlação inferiores a 0,3. Para um melhor ajuste da curva modelada à curva real, pretendemos utilizar a Modelagem Baseada em Análise Estatística. O método utilizado na obtenção da equação de ajuste da regressão é o Método dos Mínimos Quadrados (MMQ). Segundo Bucheb e 
Rodrigues (1997) apud SSI (1997), dentre os métodos de ajuste mais utilizados nos pacotes de tratamento de dados, o método dos mínimos quadrados produz as melhores estimativas da variável dependente calculada a partir de outra(s) variáveis. O emprego dos métodos de regressão citados acima é um procedimento de rotina em diversos segmentos da área de E\&P da indústria do petróleo.

Pretendemos utilizar os métodos estatísticos de regressão para modelar a variável dependente tempo de trânsito a partir de variáveis independentes, isto é, pretendemos modelar o perfil sônico a partir de outros perfis. Esse procedimento é semelhando ao proposto por Bucheb e Rodrigues (1997).

Foi visto em literatura que o coeficiente de correlação, quando utiliza-se a Modelagem Baseada em Análise Estatística, atinge valores normalmente maiores do que a modelagem baseada em equações empíricas. Os modelos utilizados adiante serão, principalmente 0 Modelo Linear Multivariável e o Modelo Não Linear Multiváriavel.

Uma visão mais avançada dessa modelagem é, após a correta validação, utilizar os coeficientes obtidos que melhor se ajustaram para gerar os perfis geofísicos em outros poços ou intervalos onde não haja a presença desse perfil.

\section{Agradecimentos}

Os autores agradecem à GEOPARK pelo fornecimento dos dados, fomento à pesquisa e viabilização de confecção deste trabalho, ao INCT-GP/CNPQ e ao CPGG/UFBA pelo apoio para a realização deste trabalho e ao apoio do Professor Geraldo Girão Nery da UFBA.

\section{Referências}

Bomfim, G. M. C. (2011) Modelagem de tempos de trânsito sintéticos em poços do campo de Quiambina, Bacia do Recôncavo, Trabalho de Graduação, Universidade Federal da Bahia, Salvador, BA.

Bomfim, G. M. C. el al. (2012) Perfis sintéticos em campos com acumulações marginais de hidrocarbonetos, Resumo expandido, V Simpósio Brasileiro de Geofísica, Salvador, BA.

Bucheb, J. A. e Rodrigues, F. S. (1997) Aspectos práticos relativos ao procedimento de geração de curvas sintéticas de perfis por meio de regressão, Boletim de Geociências da Petrobras, 11:33-47.

Gardner, G. H. F.; Gardner, L. W. e Gregory, A. R. (1974) Formation velocity and density; the diagnostic basics for stratigraphic traps., Geophysics, 39:770\{780.

Mavko, G.; Mukerji, T. e Dvorkin, J. (2009) The Rock Physics Handbook: Tools for Seismic Analysis of Porous Media, Cambridge University Press, 20 edic..

Nery, G. G. (2009) Perfilagem geofísica de poços, In: Hidrogeologia - Conceitos e Aplicações, cap. 6.2, CPRM, $3^{\text {a }}$ edic..
Pinto, R. F. (2011) Modelagem de tempos de trânsito sintéticos em poços do campo de Bela Vista, Bacia do Recôncavo, Trabalho de Graduação, Universidade Federal da Bahia, Salvador, BA.

Raymer, L. L.; Hunt, E. R. e Gardner, J. S. (1980) An improved sonic transit time-to-porosity transform, Trans. Soc. Prof. Well Log Analysts, 21st Annual Logging Symposium.

Santos, R. A. (2010) Interpretação e modelagem de perfis geofísicos de poço em campos marginais, Trabalho de graduação, Universidade Federal da Bahia, Salvador, BA.

Wyllie, M. R. J.; Gregory, A. R. e Gardner, L. W. (1956) Elastic wave velocities in heterogeneous and porous media, Geophysics, 21(1):41\{70. 